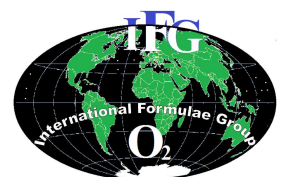

Available online at http://ajol.info/index.php/ijbes

Int. J. Biol. Chem. Sci. 8(2): 566-578, April 2014

ISSN 1997-342X (Online), ISSN 1991-8631 (Print)
International Journal

of Biological and

Chemical Sciences

Original Paper

http://indexmedicus.afro.who.int

\title{
Récente recomposition des populations de tiques prévalent en Côte d'Ivoire
}

\author{
Alassane TOURE ${ }^{1}$, Claude Aime DIAHA ${ }^{1}$, Idrissa SYLLA $^{2 *}$ et Koffi KOUAKOU ${ }^{3}$ \\ ${ }^{1}$ Laboratoire National d'Appui au Développement Agricole (LANADA)/ Laboratoire \\ Central Vétérinaire de Bingerville (LCVB), Service de Parasitologie. BP 206. Bingerville. \\ Tel: 00225224031 36, Fax: 00225224031 37. Côte d'Ivoire. \\ 2 Unité Pédagogique et de Recherche de Biologie et Physiologie Animale, \\ Université Jean Lorougnon GUEDE, Daloa. BP 150. Côte d'Ivoire. \\ 3 Laboratoire de Physiologie Animale, Université Félix Houphouët Boigny, Abidjan. \\ 22 BP 582 Abidjan 22. Côte d'Ivoire. \\ *Auteur correspondant, E-mail: syllaidris@gmail.com
}

\section{RESUME}

Une étude transversale a été conduite dans 5 villes des régions centre, est, ouest et nord de la Côte d'Ivoire au cours des mois de septembre, octobre et novembre 2011. Le but était de savoir si Rhipicephalus (Boophilus) microplus était présente dans d'autres régions hormis le sud-ouest, et la nouvelle composition des populations de tiques infestant les bovins dans ces régions. Au cours de cette étude, des tiques ont été récoltées sur un échantillon de 6 animaux dans chacun des 25 élevages répartis dans les quatre régions: centre, ouest, est et nord. Six espèces de tiques appartenant à trois genres ont été identifiées. Ce sont: Amblyomma variegatum (toutes les régions) ; Rhipicephalus (Boophilus) microplus (toutes les régions), Rhipicephalus (Boophilus) decoloratus (région est seulement), Hyalomma truncatum (régions nord, est et ouest), Rhipicephalus sanguineus (centre et nord) et Rhipicephalus senegalensis (région centre seulement). L'espèce la plus dominante, observée pendant la période d'étude, a été Rhipicephalus (Boophilus) microplus. En effet, elle est non seulement présente dans toutes les régions, mais aussi majoritaire. La répartition géographique de ces espèces de tiques, parasites des bovins, a donc été établie.

(C) 2014 International Formulae Group. All rights reserved.

Mots clés : Tiques, bovin, régions, Côte d'Ivoire.

\section{INTRODUCTION}

Les tiques sont des arthropodes hématophages obligatoires qui parasitent, pour leur repas sanguin, toutes les classes de vertébrés dans presque toutes les régions du globe et notamment en Afrique. 907 espèces sont répertoriées dans le monde, dont 223 en Afrique (Socolovschi et al., 2008).

Ces arthropodes, vecteurs d'une variété d'agents pathogènes aux animaux et aux humains (virus, bactéries et protozoaires) (Bowman et al., 2008), représentent les plus importants ectoparasites des bovins. Environ 
$80 \%$ des bovins à l'échelle mondiale sont infestés par les tiques. Elles constituent à cet égard un frein au développement de l'élevage dans plusieurs pays et une source de perte et donc de pauvreté des petits éleveurs (Bowman et al., 2008).

La lutte contre ces parasites nécessite alors non seulement l'identification des espèces qui composent leur population en milieu d'élevage, mais aussi et surtout la connaissance des agents pathogènes véhiculés par ces tiques à l'effet de rendre plus efficients les moyens de lutte.

Suite à la découverte pour la première fois du vecteur Rhipicephalus (Boophilus) microplus dans le sud-ouest de la Côte d'Ivoire (Madder et al., 2007), deux autres publications scientifiques (Madder et al., 2011 ; Toure et al., 2012) ont confirmé cette présence et les capacités d'invasion dans la même région du sud-ouest. En conséquence, la présente étude a pour objectif non seulement de savoir si Rhipicephalus (Boophilus) microplus est présente dans les autres régions, mais aussi de mettre à jour nos connaissances sur les espèces de tiques des bovins présentes dans les autres principales régions de la Côte d'Ivoire. Les départements de Korhogo, et Ferkessédougou (pour la région $\mathrm{du}$ nord), Yamoussoukro (pour la région du centre), Abengourou (pour la région de l'est) et Danané (pour la région de l'ouest) ont fait l'objet de nos investigations.

\section{MATERIEL ET METHODES \\ Zones d'étude}

Les travaux ont été effectués dans plusieurs villes appartenant aux régions centre, est, ouest et nord de la Côte d'Ivoire. Ce sont: Korhogo, et Ferkessédougou au nord, Danané à l'ouest, Yamoussoukro au centre et Abengourou à l'est. Par ailleurs, ces villes sont des chefs lieux de département ou de région (Figure 1).

Ces différentes localités sont couvertes par deux grandes zones climatiques (le climat guinéen et soudanien). Le climat guinéen de type tropical humide qui s'étend sur la zone forestière et le sud de la région des savanes (au nord) est caractérisé par des amplitudes aux températures importantes (pourcentage d'humidité atteignant $70 \%$ et des pluies allant de $1200 \mathrm{~mm}$ à Bouaké et $2300 \mathrm{~mm}$ à Danané). Cette zone connaît une saison de grandes pluies de juin à octobre, une saison de petites pluies de mars à mai, une petite saison sèche de juillet à août. Le climat soudanien ou de type soudanais s'étend sur la zone des savanes à l'exclusion de sa partie méridionale. Il est caractérisé par des amplitudes thermiques quotidiennes et annuelles relativement importantes, une humidité relativement inférieure à celle du sud du pays entre 40 et $50 \%$, et par la présence intermittente d'un vent froid et sec: l'harmattan entre décembre et février. Cette zone qui couvre Korhogo et Ferkessédougou, connaît deux saisons : une saison de pluies à partir d'avril et une saison sèche de novembre à mars avec quelques pluies de juillet à octobre.

\section{Prélèvement, conservation et identification}

Dans les départements susvisés, au cours de la période couvrant les mois de septembre, octobre et novembre 2011, des prélèvements de tiques ont été effectués au sein de vingt cinq (25) stations d'élevages type traditionnel, ayant au moins un effectif de six (6) têtes quel que soit l'âge des bovins. Sur chacun de ces sites, les tiques ont été prélevées sur toute la surface des bovins à l'aide de pince à dents mousse en veillant à préserver leur intégrité. Un échantillon de Six bovins visiblement les plus infestés, était concerné quel que soit l'effectif. Les bœufs, après contention de l'animal, ont été prélevés pendant environ 15 minutes. Deux départements (Korhogo et Ferkessédougou) ont été choisis dans le nord car la région du nord a toujours été la zone du pays ayant la plus forte activité d'élevage. Pour les autres régions, nous avons donc choisi un seul département où l'activité d'élevage est la plus forte. Dans chaque département, quatre fermes tirées au hasard à partir du groupement des éleveurs ont été échantillonnés exceptés pour 
l'ouest et le centre où sept (7) et six (6) fermes ont été respectivement choisies aléatoirement de la même façon. Pour éviter les facteurs de confusions, les fermes du même village ont été considérées comme une ferme unique (Tableau 1).

Les tiques récoltées, ont ensuite été conservées dans l'éthanol à $70{ }^{\circ} \mathrm{C}$ dans des flacons en plastic dur et identifié (Numéro de ferme, sexe, âge, race, sain ou malade, importé ou né sur la ferme) pour chaque animal. Nous avons recueilli des informations complémentaires sur chacune des fermes enquêtées incluant d'éventuels problèmes avec les tiques ou l'usage des acaricides.

L'identification au stéréoscope ainsi qu'au microscope optique des différentes espèces de tiques au laboratoire s'est basée sur leurs caractéristiques morpho-anatomiques selon Walker et al. (2003).

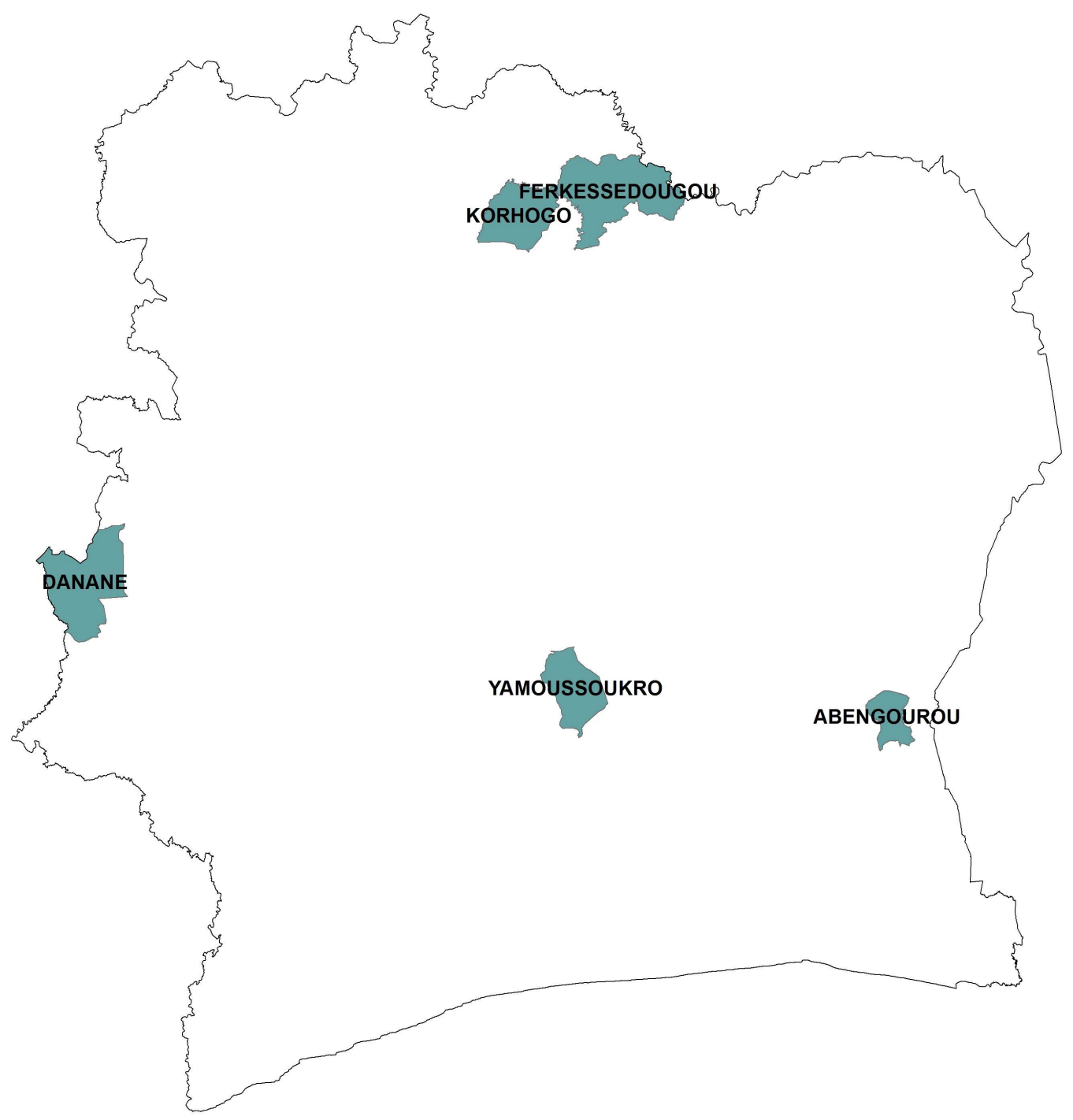

Figure 1 : Carte de localisation des zones d'étude. Source : BNTED/Centre de Cartographie et de Télédétection. 


\section{RESULTATS}

\section{Effectif de tiques récoltées par région d'étude}

Nous avons noté pour chacune des régions les effectifs suivants :

Région de Yamoussoukro: 959 tiques prélevées dans 06 fermes prospectées ;

Région de Korhogo: 04 fermes prospectées pour 635 tiques prélevées ;

Région de Ferkessédougou: 04 fermes visitées pour 570 tiques prélevées ;

Région d'Abengourou : 613 tiques prélevées sur 04 fermes ;

Région de Danané : 154 tiques prélevées sur 7 fermes prospectées ;

$\mathrm{Au}$ total, 2931 tiques ont été récoltées au cours de cette étude.

\section{Espèces de tiques identifiées}

Le résultat de la diagnose au laboratoire a révélé la présence de 06 espèces de tiques rattachées à 04 genres ou sous genre. Il s'agit de:

- Amblyomma spp (26.8\%);

- Rhipicephalus spp $(0.5 \%)$;

- Rhipicephalus (Boophilus)spp (70.9\%) et

- Hyalomma spp (1.8\%).

L'effectif et les proportions de tiques par genre et par région sont consignés dans les Tableaux 2 à 6 (Effectif et proportion des tiques récoltées par genre et par région).

Il ressort des Tableaux 2 à 6 que :

ÐDans la région de Ferkessédougou, des 570 tiques récoltées, il y a 498 tiques du sous genre Rhipicephalus (Boophilus), 58 du genre Amblyomma et 14 du genre Hyalomma (Tableau 2).

ÐDans la région de Korhogo, sur un total de 635 tiques récoltées issues de trois genres, 542 concernent le sous genre Rhipicephalus (Boophilus), 57 concernent le genre Amblyomma, 34 tiques sont du genre Hyalomma et seulement 02 représentent le genre Rhipicephalus (Tableau 3).

ЭPour ce qui est de la région d'Abengourou, 613 tiques au total ont été récoltées dont 447 du sous genre Rhipicephalus (Boophilus), 164 $\mathrm{du}$ genre Amblyomma et 02 du genre Hyalomma (Tableau 4).
ODans la région de Yamoussoukro, 959 tiques au total et appartenant à trois genres ont été récoltées: $487 \mathrm{du}$ sous genre Rhipicephalus (Boophilus), $458 \mathrm{du}$ genre Amblyomma et 14 du genre Rhipicephalus (Tableau 5).

ӘEnfin, au niveau de la région de Danané, nous avons obtenu 103 tiques du sous genre Rhipicephalus(Boophilus), $43 \mathrm{du}$ genre Amblyomma et 2 du genre Hyalomma (Tableau 6).

Par ailleurs, les espèces associées à ces genres, identifiées en se basant sur leurs caractéristiques morpho-anatomiques sont les suivantes :

$$
\begin{array}{ll}
- & \text { Amblyomma variegatum }(26.8 \%) ; \\
- & R \text { (Boophilus) decoloratus }(3.2 \%) ; \\
- & R \text { (Boophilus) microplus }(67.7 \%) ; \\
- & \text { Hyalomma truncatum }(1.8 \%) ; \\
- & \text { Rhipicephalus sanguineus }(0.5 \%) ; \\
- & \text { Rhipicephalus senegalensis }(0.1 \%) ;
\end{array}
$$

La proportion de ces espèces de tiques par région d'étude est présentée dans la Figure 2 résultante du Tableau 7 .

Ces résultats montrent aussi que toutes les tiques appartenant au genre Amblyomma sont toutes de la même espèce, en l'occurrence variegatum. En ce qui concerne le genre Rhipicephalus, il y a plusieurs espèces. L'importance de ces espèces à l'intérieur du genre Rhipicephalus est très variable. Il y 4 espèces représentées: decoloratus, microplus, sanguineus, et senegalensis.

Le genre Hyalomma est globalement minoritaire et n'est représenté que par une seule espèce à savoir truncatum.

Dans toutes les régions y compris toutes les fermes, Rhipicephalus Boophilus microplus est très majoritaire par rapport à toutes les autres espèces de tiques. Amblyomma variegatum vient en seconde position de façon constante dans chacune de ces régions y compris dans chacune des fermes (Figue 2).

Cette étude nous a permis d'établir une carte régionale présentant la répartition géographique des espèces de tiques parasitant les bovins (Figure 3). 
A. TOURE et al. / Int. J. Biol. Chem. Sci. 8(2): 566-578, 2014

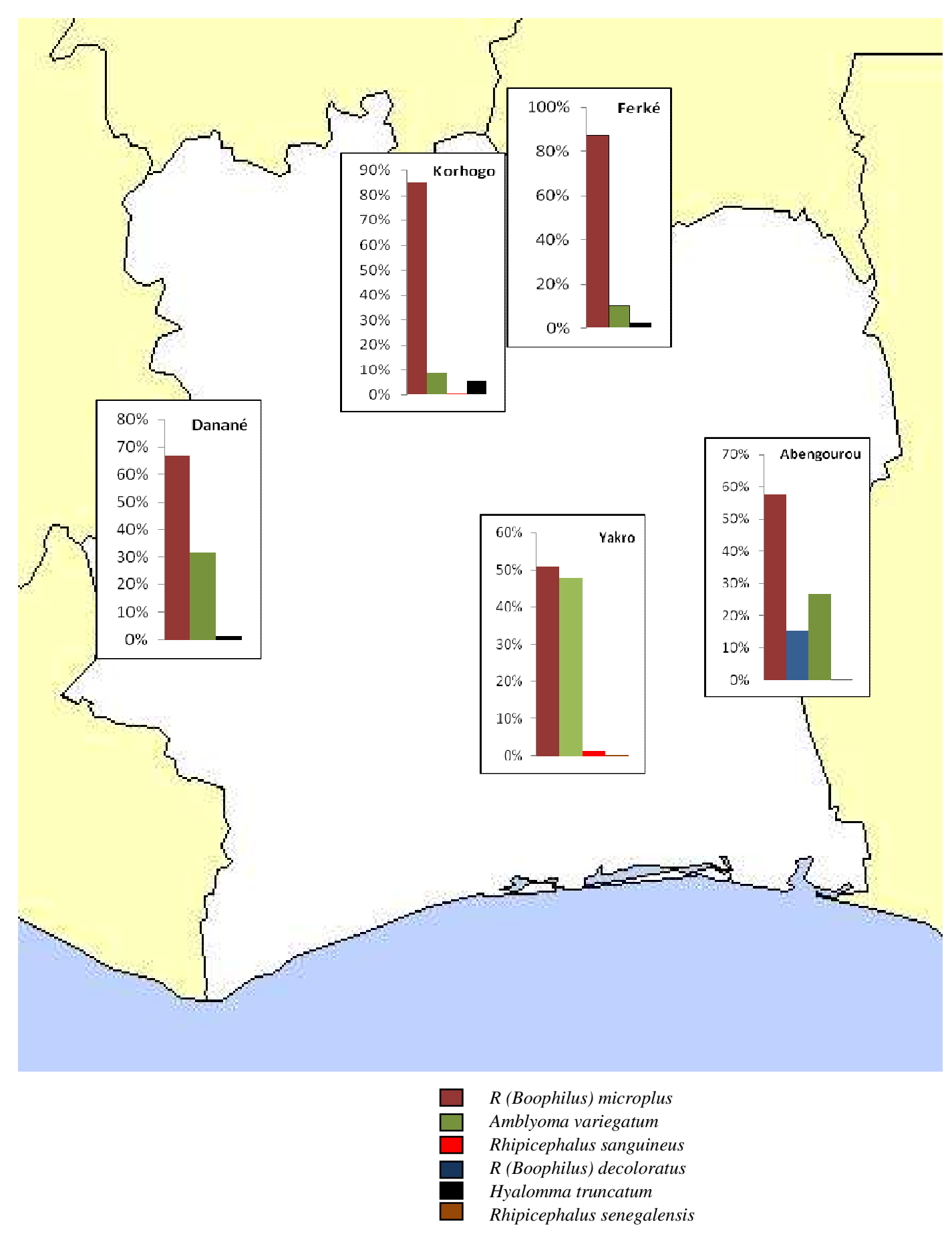

Figure 2 : Importances relatives des espèces de tiques rencontrées par région. 

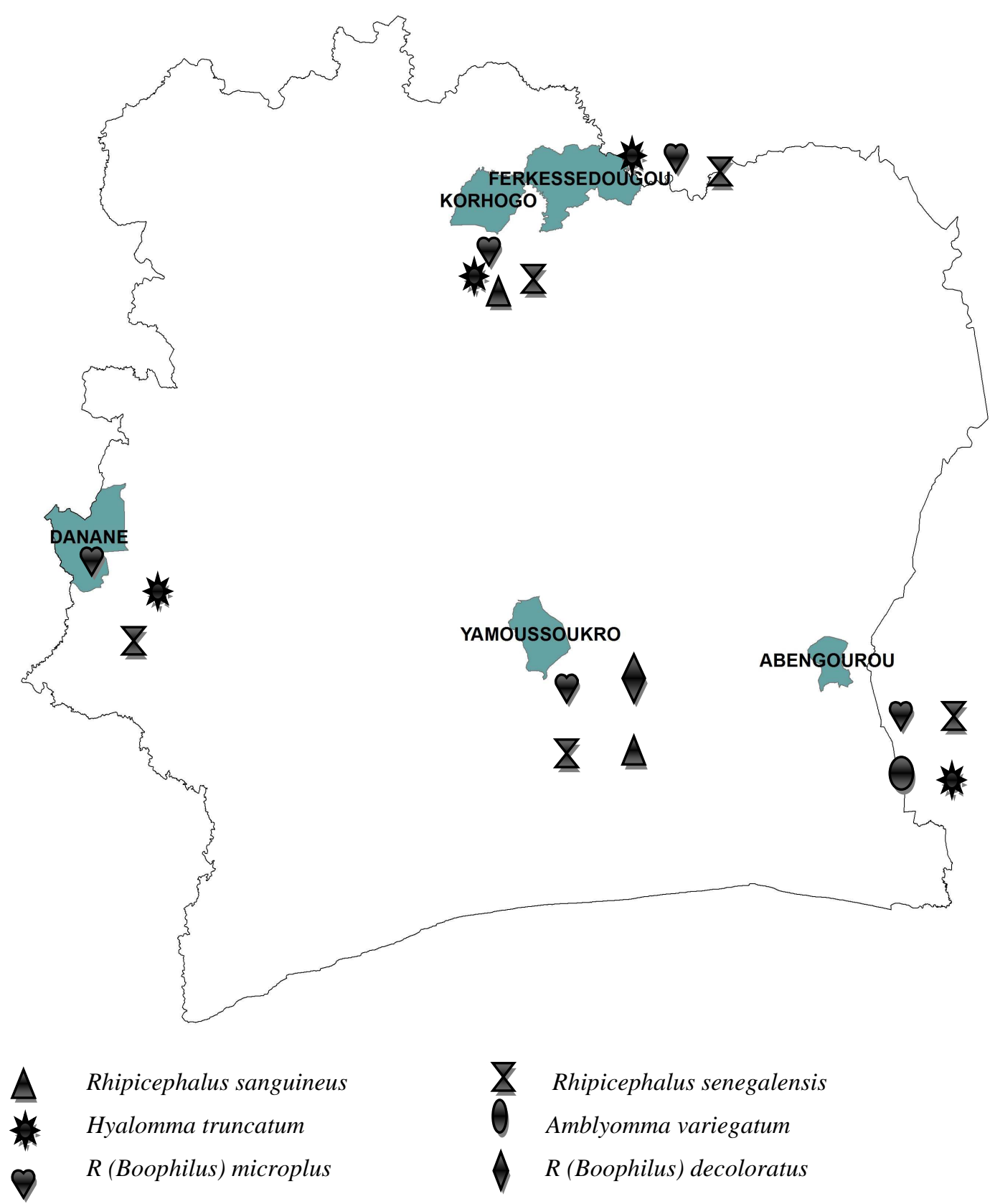

Figure 3 : Répartition géographique des espèces de tiques parasitant les bovins dans les cinq régions étudiées. 
Tableau 1: Localisation des coordonnées GPS et l'agenda des prélèvements.

\begin{tabular}{|c|c|c|c|c|}
\hline \multirow[t]{2}{*}{ Fermes } & \multicolumn{2}{|c|}{ Géo-références } & \multirow{2}{*}{$\begin{array}{c}\text { Effectifs } \\
\text { du troupeau }\end{array}$} & \multirow{2}{*}{$\begin{array}{c}\text { Dates de } \\
\text { prélèvement }\end{array}$} \\
\hline & Longitudes & Latitudes & & \\
\hline 1 & $-4,96154$ & 6,50231 & 225 & $19 / 09 / 2011$ \\
\hline 2 & $-5,52280$ & 6,91029 & 208 & $20 / 09 / 2011$ \\
\hline 3 & $-5,34485$ & 6,92816 & 35 & $20 / 09 / 2011$ \\
\hline 4 & $-5,34487$ & 6,92816 & 49 & $20 / 09 / 2011$ \\
\hline 5 & $-5,36047$ & 6,89388 & 59 & $20 / 09 / 2011$ \\
\hline 6 & $-5,15463$ & 6,75183 & 41 & $21 / 09 / 2011$ \\
\hline 7 & $-5,58342$ & 9,24785 & 98 & $04 / 10 / 2011$ \\
\hline 8 & $-5,51541$ & 9,43985 & 186 & $04 / 10 / 2011$ \\
\hline 9 & $-5,74637$ & 9,29951 & 112 & 05/10/2011 \\
\hline 10 & $-5,76616$ & 9,05735 & 82 & 05/10/2011 \\
\hline 11 & $-5,15844$ & 9,73908 & 103 & $07 / 10 / 2011$ \\
\hline 12 & $-5,22632$ & 9,37529 & 93 & $07 / 10 / 2011$ \\
\hline 13 & $-5,12383$ & 9,58539 & 106 & 08/10/2011 \\
\hline 14 & $-5,12454$ & 9,58564 & 114 & 08/10/2011 \\
\hline 15 & $-8,21806$ & 7,13844 & 52 & $08 / 11 / 2011$ \\
\hline 16 & $-8,22302$ & 7,34401 & 36 & $08 / 11 / 2011$ \\
\hline 17 & $-8,03721$ & 7,2886 & 45 & $09 / 11 / 2011$ \\
\hline 18 & $-8,26514$ & 7,19344 & 23 & $09 / 11 / 2011$ \\
\hline 19 & $-8,14655$ & 7,39099 & 27 & $10 / 11 / 2011$ \\
\hline 20 & $-8,28308$ & 7,27268 & 32 & $10 / 11 / 2011$ \\
\hline 21 & $-8,11738$ & 7,22083 & 18 & $21 / 11 / 2011$ \\
\hline 22 & $-3,47390$ & 6,72837 & 97 & $23 / 03 / 2012$ \\
\hline 23 & $-3,47390$ & 6,72837 & 89 & $23 / 03 / 2012$ \\
\hline 24 & $-3,50264$ & 6,71371 & 235 & $24 / 03 / 2012$ \\
\hline 25 & $-3,51061$ & 6,76838 & 169 & $24 / 03 / 2012$ \\
\hline
\end{tabular}


Tableau 2 : Population des tiques récoltées (Région de Ferkessédougou).

\begin{tabular}{lccccc}
\hline $\mathbf{N}^{\circ}$ de la ferme & Amblyomma & $\begin{array}{c}\text { Rhipicephalus } \\
\text { (Boophilus) }\end{array}$ & Rhipicephalus & Hyalomma & Total \\
\hline Ferme 11 & 10 & 137 & 0 & 0 & 147 \\
Ferme 12 & 9 & 102 & 0 & 0 & 111 \\
Ferme 13 & 18 & 128 & 0 & 8 & 154 \\
Ferme 14 & 21 & 131 & 0 & 6 & 158 \\
Total & 58 & 498 & 0 & 14 & 570 \\
Proportion & $\mathbf{1 0 , 2 \%}$ & $\mathbf{8 7 , 4 \%}$ & $\mathbf{0 , 0 \%}$ & $\mathbf{2 , 5 \%}$ & $\mathbf{1 0 0 \%}$ \\
\hline
\end{tabular}

Tableau 3: Population des tiques récoltées (Région de Korhogo).

\begin{tabular}{lccccc}
\hline $\begin{array}{l}\mathbf{N}^{\circ} \text { de la } \\
\text { ferme }\end{array}$ & Amblyomma & $\begin{array}{c}\text { Rhipicephalus } \\
\text { (Boophilus) }\end{array}$ & Rhipicephalus & Hyalomma & Total \\
\hline Ferme 7 & 22 & 183 & 2 & 15 & 222 \\
Ferme 8 & 19 & 205 & 0 & 19 & 243 \\
Ferme 9 & 0 & 47 & 0 & 0 & 47 \\
Ferme 10 & 16 & 107 & 0 & 0 & 123 \\
Total & 57 & 542 & 2 & 34 & 635 \\
Proportion & $\mathbf{9 , 0 \%}$ & $\mathbf{8 5 , 4 \%}$ & $\mathbf{0 , 3 \%}$ & $\mathbf{5 , 4 \%}$ & $\mathbf{1 0 0 \%}$ \\
\hline
\end{tabular}

Tableau 4: Population des tiques récoltées (Région d'Abengourou).

\begin{tabular}{lccccc}
\hline N' de la ferme & Amblyomma & $\begin{array}{c}\text { Rhipicephalus } \\
\text { (Boophilus) }\end{array}$ & Rhipicephalus & Hyalomma & Total \\
\hline Ferme 22 & 32 & 29 & 0 & 0 & 61 \\
Ferme 23 & 46 & 41 & 0 & 0 & 87 \\
Ferme 24 & 29 & 96 & 0 & 0 & 125 \\
Ferme 25 & 57 & 281 & 0 & 2 & 340 \\
Total & 164 & 447 & 0 & 2 & 613 \\
Proportion & $\mathbf{2 6 , 8 \%}$ & $\mathbf{7 2 , 9 \%}$ & $\mathbf{0 , 0 \%}$ & $\mathbf{0 , 3 \%}$ & $\mathbf{1 0 0 \%}$ \\
\hline
\end{tabular}


Tableau 5: Population des tiques récoltées (Région de Yamoussoukro).

\begin{tabular}{lccccc}
\hline $\mathbf{N}^{\circ}$ de la ferme & Amblyomma & $\begin{array}{c}\text { Rhipicephalus } \\
\text { (Boophilus) }\end{array}$ & Rhipicephalus & Hyalomma & Total \\
\hline Ferme 1 & 102 & 109 & 7 & 0 & 218 \\
Ferme 2 & 9 & 15 & 0 & 0 & 24 \\
Ferme & 107 & 123 & 2 & 0 & 232 \\
Ferme 4 & 115 & 119 & 5 & 0 & 239 \\
Ferme 5 & 28 & 4 & 0 & 0 & 32 \\
Ferme 6 & 97 & 117 & 0 & 0 & 214 \\
Total & 458 & 487 & 14 & 0 & 959 \\
Proportion & $\mathbf{4 7 , 8 \%}$ & $\mathbf{5 0 , 8 \%}$ & $\mathbf{1 , 5 \%}$ & $\mathbf{0 , 0 \%}$ & $\mathbf{1 0 0 \%}$ \\
\hline
\end{tabular}

Tableau 6: Population des tiques récoltées (Région de Danané).

\begin{tabular}{lccccc}
\hline $\mathbf{N}^{\circ}$ de la ferme & Amblyomma & $\begin{array}{c}\text { Rhipicephalus } \\
\text { (Boophilus) }\end{array}$ & Rhipicephalus & Hyalomma & Total \\
\hline Ferme 15 & 18 & 28 & 0 & 0 & 46 \\
Ferme 16 & 11 & 31 & 0 & 0 & 42 \\
Ferme 17 & 17 & 30 & 0 & 0 & 47 \\
Ferme 18 & 1 & 5 & 0 & 2 & 8 \\
Ferme 19 & 1 & 1 & 0 & 0 & 2 \\
Ferme 20 & 0 & 3 & 0 & 0 & 3 \\
Ferme 21 & 1 & 5 & 0 & 0 & 6 \\
Total & 49 & 103 & 0 & 2 & 154 \\
Proportion & $\mathbf{3 1 , 8 \%}$ & $\mathbf{6 6 , 9 \%}$ & $\mathbf{0 , 0 \%}$ & $\mathbf{1 , 3 \%}$ & $\mathbf{1 0 0 , 0 \%}$ \\
\hline
\end{tabular}

Tableau 7: Importance relative des différentes espèces de tiques selon les départements.

\begin{tabular}{lccccccccccc}
\hline \multirow{2}{*}{ Espèces } & \multicolumn{3}{c}{ Yakro } & \multicolumn{3}{c}{ Abengourou } & \multicolumn{1}{c}{ Danane } & \multicolumn{3}{c}{ Korhogo } & \multicolumn{2}{c}{ Ferké } \\
\cline { 2 - 13 } & Nbre & $\mathbf{\%}$ & Nbre & $\mathbf{\%}$ & Nbre & \% & Nbre & \% & Nbre & \% \\
\hline Rhipicephalus (B) microplus & 487 & 50,8 & 354 & 57,7 & 103 & 66,9 & 542 & 85,4 & 498 & 87,4 \\
Rhipicephalus (B) decoloratus & 0 & 0,0 & 93 & 15,2 & 0 & 0,0 & 0 & 0,0 & 0 & 0,0 \\
Amblyomma variegatum & 458 & 47,8 & 164 & 26,8 & 49 & 31,8 & 57 & 9,0 & 58 & 10,2 \\
Rhipichephalus sanguineus & 12 & 1,3 & 0 & 0,0 & $\mathrm{O}$ & 0,0 & 2 & 0,3 & $\mathrm{O}$ & 0,0 \\
Rhipicephalus senegalensis & 2 & 0,2 & 0 & 0,0 & 0 & 0,0 & 0 & 0,0 & 0 & 0,0 \\
Hyalomma truncatum & 0 & 0,0 & 2 & 0,3 & 2 & 1,3 & 34 & 5,4 & 14 & 2,5 \\
TOTAL & 959 & 100 & 613 & 100 & 154 & 100 & 635 & 100 & 570 & 100 \\
\hline$\quad$ Yakro = Yamoussoukro & & & & & & & & & &
\end{tabular}

Ferké $=$ Ferkessedougou 


\section{DISCUSSION}

L'inventaire des tiques dans les différentes zones d'étude de la Côte d'Ivoire a montré que les espèces récoltées appartiennent aux trois genres Amblyomma, Hyalomma, et Rhipicephalus. Ces genres ont également été mis en évidence par Achi et al. (2001) dans le nord de la Côte d'Ivoire. Il y a au total six (06) espèces de tiques prélevées dans les troupeaux examinés. Cependant, les travaux de Knopf et al. (2002) ont inventorié dans la région de Toumodi (au centre) cinq (5) espèces dans l'ordre d'importance: Amblyomma variegatum, Rhipicephalus (Boophilus) geiygi, Rhipicephalus (Boophilus) annulatus, Rhipicephalus sanguineus, Hyalomma rufipes. Enfin, ils ont noté que Amblyomma était la tique majoritaire à travers toutes les saisons. Il faut relever que plutard, au sud-ouest de la Côte d'Ivoire en 2011, Madder et al. (2011) avaient trouvé en très faible proportion (moins de 5\% pour Rhipicephalus (B) annulatus et Rhipicephalus (B) geigyi moins de 5\%) et en 2012, Toure et al. (2012) n'avaient plus retrouver Rhipicephalus (B) annulatus et Rhipicephalus (B) geigyi.

Dans notre étude, les espèces de tiques les plus dominantes au sein des élevages enquêtés sont Rhipicephalus (Boophilus) microplus suivie de Amblyomma variegatum.

Cette étude nous a montré que sur les 2931 tiques récoltées, le sous genre Rhipicephalus (Boophilus) est le plus dominant chez ces Ixodidae infestant les bovins dans ces différentes zones. Ce sous genre comprend ici l'espèce $R$ (Boophilus) microplus $(67.7 \%)$ et l'espèce $R$ (Boophilus) decoloratus $(3.2 \%)$, et représente à lui seul (70.9\%) de la totalité des tiques récoltées. Il y a donc une recomposition profonde des genres de tique actuellement. Des études ultérieures sont pertinentes pour évaluer cette tendance dans chacune de ces régions: centre, nord, est et ouest.

Signalons que c'est la première fois qu'est récoltée l'espèce Rhipicephalus (Boophilus) microplus dans chacune de ces cinq régions: Yamoussoukro, Korhogo, Ferkessédougou, Abengourou et Danané. Cette espèce avait été découverte pour la première fois en Côte d'Ivoire dans la région d'Azaguié (sud-est) (Madder et al., 2007). Les fermes du projet laitier sud étaient concernées par cette infestation. Cette situation est vraisemblablement due à des importations non contrôlées de bovins en provenance de continents infestés.

Ensuite, Madder et al. (2011), dans la même région d'Azaguié, ont mis en évidence les capacités invasives et de remplacement des autres espèces par Rhipicephalus (Boophilus) microplus: $R$ (B) microplus (96\%), $R(B)$ decoloratus (1.5\%), $R(B)$ geigy $(2.5 \%)$.

En plus de relever pour la première fois cette espèce de tique dans d'autres régions de la Côte d'ivoire, notre étude montre également comme Madder et al. (2011) et Toure et al. (2012), la capacité d'invasion et de remplacement des autres espèces par Rhipicephalus (Boophilus) microplus. Le sous genre Rhipicephalus (Boophilus) était constitué exclusivement de l'espèce microplus. A l'exception de la région d'Abengourou (à l'est) où nous avons observé deux espèces du sous genre Rhipicephalus (Boophilus): ce sont $R$ (B) microplus $(79.19 \%)$ et $R$ (B) decoloratus (20.81\%).

Ainsi, nous remarquons que Rhipicephalus (Boophilus) microplus a supplanté les autres espèces par sa prolifération car les autres espèces de Rhipicephalus (Boophilus) ont été totalement ou presque remplacées par Rhipicephalus (Boophilus) microplus. En Afrique de l'ouest, précisément au Bénin où $R$. microplus a été aussi récemment introduite comme en Côte d'Ivoire (Madder et al., 2012), l'étude de De Clercq et al. (2012) a montré qu'il y a une nette séparation géographique entre $R$. microplus et les autres espèces autochtones de Ripicephalus (geigyi, annulatus, et decoloratus): $R$ microplus est surtout présente au sud et modérément au centre alors que les 
autres espèces autochtones sont principalement au nord et modérément au centre. Il n'y a pas une telle séparation en Côte d'Ivoire bien que la Côte d'Ivoire et le Benin soient globalement situés entre les mêmes parallèles du point de vue géographique et relativement proches en Afrique de l'ouest.

Rhipicephalus (Boophilus) microplus semble parfaitement réussir la stratégie de colonisation et d'occupation des espaces favorables (Barré, 2010). Cette tendance de remplacement est d'autant plus forte que les dernières études publiées en 2002 (Knopf et al., 2002) et avant l'introduction de cette tique, n'observaient pas la présence de Rhipicephalus (Boophilus) microplus. En outre, nous pouvons raisonnablement envisager une expansion continue de cette espèce car Estrada-Pena et al. (2006) ont démontré que contrairement à Rhipicephalus (Boophilus) microplus du continent américain qui exige des niveaux de précipitation élevées pour proliférer, l'espèce homologue d'Afrique n'a besoin que de niveaux de précipitations faibles (entre mai et octobre) pour envahir davantage une zone. Ces niveaux de précipitations faibles existent en zone centre et nord pendant la saison sèche. Les autres régions du pays : est, ouest, centre et sud sont des zones beaucoup plus humides. En conséquence, les conditions climatiques sont très largement favorables pour Rhipicephalus (Boophilus) microplus au point qu'elle remplace au moins efficacement les autres espèces de son sous genre. La rapidité du remplacement que notre étude met en lumière est consistante avec les travaux de Tonnesen et al. (2004) dans la province du Limpopo en Afrique du sud de 1999 à 2001. Dans cette étude, le remplacement de Rhipicephalus (Boophilus) decoloratus par Rhipicephalus (Boophilus) microplus était quasi complet dans les fermes traditionnelles. Sur la même tendance pendant la même période, on est passée de $56,8 \%$ à $25,1 \%$ de fermes commerciales infestées par $R$ (Boophilus) decoloratus. Par ailleurs, Sutherst (1987) renforce nos données car il fait une estimation de la durée du remplacement de 1 à 3 ans, avec l'outil de la modélisation.

Notons que la résistance acquise par cette espèce à la plupart des acaricides disponibles sur le marché pourrait avoir favorisé le remplacement des autres espèces de Rhipicephalus (Boophilus) (Baffi et al., 2008). En effet, lors de la collecte des tiques dans les milieux d'étude, il a été remarqué que les animaux récemment traités présentaient des charges élevées de tiques vivantes notamment les Rhipicephalus (Boophilus).

Les tiques de faible occurrence se limitent à certaines régions. C'est le cas des tiques des espèces Rhipicephalus (sanguineus et senegalensis) au centre et au nord et des tiques du genre Hyalomma rencontrées dans les régions est, ouest et nord. Après les tiques du sous genre Rhipicephalus (Boophilus), les tiques de l'espèce $R$ sanguineus $(0.5 \%)$ sont les plus abondantes. Cette espèce est devenue la plus répandue dans les régions tropicales et subtropicales en raison de son tropisme particulier pour le chien domestique (Fahmy et al., 1981) qui est bien souvent utilisé pour la garde par les éleveurs. Ensuite, avec 0,1\% du total des tiques collectées Rhipicephalus senegalensis répertorié au centre, nos données concordent avec la proportion de moins de $1 \%$ trouvée au sud-ouest par Madder et al. (2011).

En ce qui concerne Hyalomma truncatum, nos travaux ont montré son caractère très minoritaire $(1,8 \%$ des tiques collectées) et présente à l'ouest, l'est et le nord. D'ailleurs, la plupart des précédentes études avaient mis en évidence ces aspects au centre (Knopf et al., 2002) et au nord de la Côte d'Ivoire (Achi et al., 2001).

\section{Conclusion}

L'étude des tiques ectoparasites des bovins dans les régions de Korhogo, Ferkéssédougou, Danané, Yamoussoukro et Abengourou a permis de conclure qu'il y a six espèces de tiques appartenant à trois 
genres. Ces espèces sont: Rhipicephalus(Boophilus) microplus, Rhipicephalus(Boophilus) decoloratus, Amblyomma variegatum, Rhipicephalus senegalensis, Rhipicephalus sanguineus, Hyalomma trucatum. En outre, pour la première fois, nous avons montré que Rhipicephalus (Boophilus) microplus fait désormais partie des populations de tiques dans le nord, l'est, le centre et l'ouest de la Côte d'Ivoire. Cette espèce y est même majoritaire en signant par ailleurs une tendance de remplacement des espèces appartenant au même sous genres.

Enfin, nos travaux ont permis d'actualiser les données sur la répartition géographique des différents genres et espèces de tiques infestant les bovins ainsi que leur importance relative.

\section{REFERENCES}

Achi L, Zinsstag J, Nagto Y, Kan Y. 2001. Impact des tiques sur les métis montbéliarde dans les fermes Modernes. Rapport d'activité Centre Suisse de Recherche Scientifique en CI, 139p.

Baffi MA, De Souza GR, De Souza CS, Ceron CR, Bonetti AM. 2008. Esterase enzymes involved in pyrethroid and organophosphate resistance in a Brazilian population of Riphicephalus (Boophilus) microplus (Acari, Ixodidae). Mol. Biochem. Parasitol., 160: 70-73.

Barré N, Uilenberg G. 2010. Propagation de parasites transportés avec leurs hôtes : cas exemplaires de deux espèces de tiques du bétail. Rev. Sci. Tech. Off. Int. Epiz., 29 (1): 135-147.

Bowman AS, Nuttall PA. 2008. Ticks Biology, Disease and Control. Cambridge University Press: Cambridge.

De Clercq EM, Vanwambeke SO, Sungirai M, Adehan S, Lokossou R, Madder M. 2012. Geographic distribution of invasive cattle tick Rhipicephalus microplus, a wide survey in Benin. Experimental and Applied Acarology, 58: 441-452.
Devos J. 2001. Babesia divergens: comparaison de l'intérêt de l'IFI et de la PCR pour la réalisation d'enquêtes épidémiologiques. Travail de fin d'année présenté pour obtenir le diplôme de Spécialisation en Production et Santé Animales Tropicales, Institut de Médecine Tropicale, Anvers.

Estrada-Pena A, Bouattour A, Camicas JL, Guglielmone A, Horak I, Jongejean F, Latif A, Pegram R, Walker AR. 2006. The known distribution and ecological preferences of tick subgenus Boophilus (Acari: Ixodidae) in Africa and latin America. Experimental and Applied Acarology, 38: 219-235.

Fahmy MAM, Arafa MS, Mandour AM, Sema AAA. 1981. Survey of hard tick (Ixodidae) infesting domestic animals in Assuit Governorate, Upper Egypt. Acta Parasitol. Polonica, 28(9): 91-96.

Farougou S, Tassou AW, Tchabode DM, Kpodekon M, Boko C, Youssao AKI. 2007. Tiques et hémoparasites du bétail dans le nord-Benin. Rev. Med. Vét., 158: (8-9): 463-467.

Gragnon BG. 2005. Prévalence de l'anaplasmose et de la babesiose bovines en zone forestière de la Côte d'Ivoire. Travail de fin d'année présenté pour l'obtention du grade de Master of Science en Santé Animale Tropicale. Institut de Médecine Tropicale, Anvers.

Katholi CR, Toé L, Merriweather A, Unnasch TR. 1995. Determining the prevalence of Onchocerca volvulus Infection in Vector Population by Polymerase Chain Reaction Screening of Black Files. The journal of Infectious Diseases, 172(5): 1414-1417.

Knopf L, Komoin-Oka C, Betshart B, Jongejan F, Gottstein B, Zinsstag J. 2002. Seasonal epidemiology of ticks and aspects of cowdriosis in N'Dama village cattle in central Guinea savannah of Côte d'Ivoire. Prev. Vet. Med., 53(1): 21-30. 
Knopf L, Komoin-Oka C, Zinsstag J. 2002. Le rôle du parasitisme dans les élevages bovins N'dama de la savane humide de la CI. Rapport d'activité Centre Suisse de Recherches Scientifique en CI, 19992000, 114p.

Lynen G, Zeman P, Bakuname C, Di Giulio G, Mtui P, Sanka P, Jongejan F. 2008. Shifts in the distributional ranges of Boophilus ticks in Tanzania: evidence that a parapatric boundary between Boophilus microplus and B. decoloratus follows climate gradients. Exp Appl Acarol., 44: 147-164.

Madder M, Thys E, Geysen D, Baudoux C, Horak I. 2007. Boophilus microplus ticks found in West Africa. Exp Appl Acarol. 43: 233-234.

Madder M, Thys E, Achi L, Toure A, De Deken R. 2011. Rhipicephalus (Boophilus) microplus: a most successful invasive tick species in WestAfrica. Exp. Appl. Acarol., 53(2): 139145

Madder M, Adehan S, De Deken R, Adehan $\mathrm{R}$, Lokossou R, 2012. New foci of $R$ microplus in west Africa. Experimental and Applied Acarology, 56: 385-390.

Morel PC. 2000. Maladies à Tiques du Bétail en Afrique. In Précis de Parasitologie Vétérinaire Tropicale. Chartier C, Itard
J, Morel PC, Troney PM (eds). TEC \& DOC: Paris; 452-644.

Morse SS. 1995. Factors in the emergence of infectious diseases. Emerging Infectious Diseases, 1(1):7-15.

Socolovschi C, Doudier B, Pages F, Parola P. 2008. Tiques et maladies transmises à l'homme en Afrique. Médecine Tropicale, pp 119-133.

Sutherst RW. 1987. The dynamics of hybrid zone between tick (Acari) species. Int. J. Parasitology, 17: 921-926.

Tonnesen MH, Penzhorn BL, Bryson NR, Stoltsz WH, Massibigiri T. 2004. Displacement of Boophilus decoloratus by Boophilus microplus in southpansberg region, Limpopo province, South Africa. Experimental and Applied Acarology, 32: 199-208.

Toure A, Komoin-Oka C, Sylla I. 2012. Cattle ticks population and prevalence of Babesia spp amongst its vector: Rhipicephalus (Boophilus) microplus in a zone of Ivory Coast. Int. J. Biol. Chem. Sci., 6(4): 1574-1581.

Walker AR, Bouatour A, Camicas JL, Estrada-Pena A, Horak IG, Latif AA, Pegram RG, Preston PM. 2003. Ticks of domestic animals in Africa: a guide to Identification of Species, Bioscience Report, 47-221. 